Commun:HEITn Communication et organisation

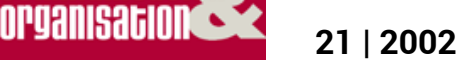

Espace et communication dans les organisations

\title{
Espaces de travail et communication - Une lecture psychosociale
}

Gustave-Nicolas Fischer et Chantal Fousse

\section{OpenEdition}

1 Journals

Édition électronique

URL : http://journals.openedition.org/communicationorganisation/2651

DOI : 10.4000/communicationorganisation.2651

ISSN : $1775-3546$

Éditeur

Presses universitaires de Bordeaux

Édition imprimée

Date de publication : 1 mai 2002

ISSN : 1168-5549

Référence électronique

Gustave-Nicolas Fischer et Chantal Fousse, «Espaces de travail et communication - Une lecture psychosociale », Communication et organisation [En ligne], 21 | 2002, mis en ligne le 27 mars 2012, consulté le 10 décembre 2020. URL : http://journals.openedition.org/communicationorganisation/ 2651 ; DOI : https://doi.org/10.4000/communicationorganisation.2651

Ce document a été généré automatiquement le 10 décembre 2020.

(c) Presses universitaires de Bordeaux 


\title{
Espaces de travail et communication - Une lecture psychosociale
}

\author{
Gustave-Nicolas Fischer et Chantal Fousse
}

\section{Introduction}

1 La question de l'impact des espaces de travail sur la satisfaction, l'efficacité et le bien-être intéresse depuis longtemps les aménageurs, les entreprises et les chercheurs.

2 Dans une perspective psychosociale, un environnement doit être considéré à travers la relation qui s'établit avec lui. C'est donc le système d'interaction espace-individu qui permet de saisir la façon dont un environnement est utilisé et perçu.

3 Une des manières d'aborder la relation espace de travail-individu, et notamment la relation espace-communication, c'est de considérer les modèles qui sous-tendent l'aménagement de l'entreprise, d'un côté, et l'organisation de la communication, de l'autre.

4 Les relations existant entre organisation et communication sont en effet déterminées par deux types de logique :

5 - La façon dont un type d'espace est conçu et organisé (espace architectural, aménagement intérieur)

6 - La façon dont un type de travail est pensé et organisé.

7 On ne peut pas séparer espace et travail car on a affaire à un système d'interdépendance. En conséquence, la relation entre espace et communication doit être examinée à deux niveaux: celui des prescriptions normatives qui définissent comment les individus doivent entrer dans le jeu de cette relation; celui des pratiques sociales qui résultent des stratégies d'usages de l'espace, notamment informel, montrant la valeur symbolique, culturelle de cette relation, qui ne peut être réduite à des propriétés purement fonctionnelles.

8 Après avoir présenté quelques éléments théoriques définissant les caractéristiques de la relation espace-communication, nous montrerons d'une part le lien entre modèle 
d'aménagement et communication, et, d'autre part, celui entre modèle du travail, particulièrement les nouvelles technologies, et la conception de l'espace.

Nous illustrerons ces données par un exemple d'application.

\section{Eléments théoriques} que les territoires rendaient possibles. Il a ainsi identifié trois types de territoires: les territoires à organisation fixe, les territoires à organisation semi-fixe et les espaces informels.

17 Les territoires à organisation fixe sont ceux dont les limites sont établies de manière rigide en vue de certaines activités. Se manifeste là une correspondance entre tels aspects de l'espace et tels comportements particuliers qui s'y trouvent rattachés.

18 Les espaces à organisation semi-fixe se définissent par l'ensemble des éléments semi-fixes qui s'y trouvent : équipements, mobilier, etc., ainsi que la relation existant entre ce type d'organisation spatiale et le comportement. Il apparait, à travers un certain nombre 
d'expériences, que les éléments de l'espace à caractère semi-fixe, suivant la façon dont ils sont disposés, aménagés, orientent et/ou contraignent les activités et les échanges.

Enfin, l'espace informel s'établit en fonction d'un certain nombre de distances que nous maintenons dans nos relations avec autrui.

D'autres recherches ont étudié l'influence de l'aménagement de divers espaces institutionnels ; il s'agit essentiellement d'études sur le terrain, telle que celle d'Osmond (1959), révélatrice d'un type d'influence de l'espace qui correspond aux éléments semifixes; il a appelé «'espaces sociofuges », des espaces qui séparent et isolent les individus les uns des autres; les espaces dits « sociopètes » étant ceux dont l'aménagement favorise et développe les échanges entre les individus.

Dans une autre perspective, des travaux ont montré que chaque espace aménagé crée des situations spécifiques en leur fixant un cadre; tout comportement est en quelque sorte façonné par un cadre spatial dans lequel il se manifeste. Barker (1968) a appelé ces aménagements des "behavior setting ", c'est-à-dire une assise topologique de nature socioculturelle à l'intérieur de laquelle se développent des conduites marquées par des interactions entre les caractéristiques physiques et les données culturelles propres à un lieu. L'intérêt du behavior setting repose sur le fait qu'un espace social consiste, d'une part, en une réalité objective ayant des composantes interdépendantes, et d'autre part, en des formes comportementales stables. Il révèle ainsi que tout environnement aménagé constitue une unité composée d'éléments physiques qui interfèrent avec des données sociales et culturelles propres aux lieux, aux contextes et aux groupes qui s'y meuvent.

Ainsi l'organisation de l'espace oriente les comportements et la communication. Si toutes nos conduites se trouvent prises dans une certaine organisation imposée par les contraintes d'un environnement, en revanche, les relations que nous pouvons développer à notre tour ne sont pas toujours le produit d'une influence spatiale définie : à l'intérieur de chaque espace existe un champ des possibles plus ou moins large qui permet d'établir sa place et d'aménager son comportement en l'adaptant à la situation socio-spatiale.

Il est important de souligner que la distance physique crée de la distance sociale. En effet, cette distance est souvent déterminée au cours des échanges par les équipements de bureau ou les positions des sièges qui maintiennent les individus dans des limites allant d'un mètre vingt à trois mètres soixante.

Tous ces éléments spatiaux (configuration, disposition, taille) jouent sur la manière dont les individus occupent un lieu et le perçoivent.

$\mathrm{Au}$ niveau organisationnel, la grille de lecture proposée par la psychologie de l'environnement permet d'appréhender deux aspects de l'aménagement, à savoir d'un côté, l'espace architectural, et de l'autre, l'aménagement intérieur, comme deux expressions spécifiques du rapport espace et communication.

Dans le premier cas, l'espace est utilisé comme vecteur de l'aménagement organisationnel et de construction de cette image et dans l'autre, l'espace est utilisé comme un élément de l'organisation du travail.

\section{L'espace architectural comme outil de communication}

Si on considère l'espace architectural sous l'angle de la communication, on observe qu'il est habituellement l'objet d'un traitement en termes de design. Les bâtiments jouent ainsi 
le rôle d'une mise en scène de l'entreprise. L'espace extérieur a une fonction de carte de visite destinée à créer une image positive de l'organisation.

Nombre d'entreprises utilisent ainsi l'espace comme un emblème ; les bureaux d'un siège social sont par exemple aménagés pour exprimer comment l'entreprise se voit et veut se donner à voir; cet espace annonce l'entreprise et constitue un instrument de représentation et de communication avec l'extérieur.

L'espace architectural est donc un des supports de l'image de l'organisation dans la mesure où il est la figure symbolique qui constitue un outil de communication à destination de l'environnement extérieur. Cet aménagement est un véhicule de la culture organisationnelle, car il constitue un message esthétique destiné plus aux partenaires de l'entreprise (fournisseurs, clients, visiteurs), qu'à ses employés.

Pour rendre compte de cette dimension, des chercheurs ont proposé la notion d'artefact symbolique». Davis (1984) appelle artefact symbolique les aspects de l'environnement physique qui orientent, individuellement ou collectivement, l'interprétation donnée à l'aménagement ; les artefacts symboliques véhiculent ainsi quatre messages principaux : la nature des activités et leurs priorités, le statut des individus (reconnaissance de la valeur personnelle et pouvoir), l'orientation vers une tâche et la qualité de l'environnement.

\section{L'aménagement intérieur comme réseau de relations}

31 La topologie de l'espace intérieur définit globalement une organisation comme un système de places (être proche ou loin, être au centre ou à la périphérie). L'espace organise et oriente la communication; il impose des voies de circulation, des canaux formels qui épousent la nécessité de structurer l'information sur un mode fonctionnel. Le dispositif spatial permet de constater que l'on ne communique pas avec qui l'on veut; en raison des positions relatives, les contraintes du milieu organisationnel orientent plus ou moins fortement les communications.

On fait croire, aujourd'hui, que la communication est une sorte de " recette universelle " d'efficacité dans la société : "plus on communique, mieux la société fonctionne». On passe ainsi du fait de la communication à une idéologie de la communication. Or, c'est une image toute différente qui doit être proposée si l'on considère les rapports entre espace et communication tels qu'ils découlent des modèles d'aménagements intérieurs concernant les espaces de bureaux.

\section{Modèles d'aménagement et communication}

33 Les concepts et formes d'aménagement liés à l'évolution de l'organisation du travail permettent de comprendre les liens existant entre le fait de configurer l'espace de telle ou telle manière et le fait de le considérer comme performant par rapport à la circulation de l'information et de la communication.

L'étude des modèles du bureau paysager et du bureau alvéolaire permet, à titre d'exemple, d'apporter un éclairage à la compréhension de cette relation. 


\section{Le bureau paysager (office landscape)}

I1 s'agit d'un grand espace ouvert dans lequel les activités sont organisées selon le principe de la fluidité des informations et de la flexibilité du lieu en vue de faciliter la communication et d'améliorer le travail. Il est caractérisé par la disparition de murs, de cloisons et de séparations. Cette conception, développée sous le terme de bureau paysager et connue pour être une idée nord-américaine, est en fait née en Allemagne; ce sont deux frères du nom de Schnelle qui lanceront cette idée; la société Quickborner Team qu'ils créeront s'installa aux États-Unis et le concept ne fut repris en Europe que dans les années soixante. Il va donner lieu à l'apparition du bureau fonctionnel considéré comme un grand espace ouvert (office landscape), sans cloisons ni portes, peuplé de personnes, de tables, de sièges, de machines, et, ultérieurement, de plantes vertes.

Le principe de ces aménagements est basé sur une idée de la communication qui associe ouverture de l'espace, productivité et disparition des indices hiérarchiques. On regroupe dans une même zone les personnes qui doivent travailler ensemble; les séparations physiques doivent donc être minimes pour créer un paysage intérieur (Burolandschaft). L'espace est également organisé de manière à réduire au maximum le stockage du papier d'un poste à un autre. Dans cette optique, l'aménagement ouvert entraîne une autre conséquence: la disposition de certains meubles correspond non seulement à une séparation des lieux, mais à une identification des territoires distincts dans la mesure où les employés perçoivent par exemple de tels artifices comme des barrières à franchir.

Ainsi la disposition du mobilier, la présence de plantes vertes, la recherche de couleur, sont autant de moyens permettant de créer un paysage intérieur qui doit fonctionner comme un système de communication tout en permettant une certaine intimité à travers la délimitation de zones fonctionnelles séparées par des écrans artificiels. L'ouverture d'un espace est dans une telle optique associée à une meilleure communication, car l'espace est perçu comme servant directement de support et de cadre à l'organisation des activités.

En outre, ce modèle s'inspire d'un principe fondamental qui est celui de la Kommunikationsfähigkeit de l'espace conçu par les créateurs du bureau paysager, à savoir le fait que l'espace ouvert est en soi un facilitateur de la communication. En d'autres termes, on attribue au système d'ouverture de l'espace des vertus non seulement de facilitation de la circulation des informations, mais également de stimulation des interactions, du fait qu'il favoriserait notamment le partage d'informations significatives et qu'il aurait de surcroît un rôle support dans le règlement des conflits interpersonnels.

Dans le bureau paysager, c'est la configuration de l'espace ouvert qui est considérée comme un outil de communication. On a affaire à une hypothèse implicite des aménageurs et des décideurs, qui établissent une relation de causalité entre ouverture et communication, d'une part, et entre aménagement ouvert et motivation, d'autre part.

En d'autres termes, l'absence de cloisons est interprétée comme un système incitateur facilitant les échanges entre les individus. Quel est véritablement l'impact de cet aménagement sur la communication?

Certaines enquêtes ont montré qu'environ $80 \%$ de la communication dans un bureau paysager se limitaient aux échanges à l'intérieur de son propre groupe et seulement $20 \%$ s'effectuent entre les différents groupes. 
autre part, si le bureau paysager a été considéré comme une amélioration par rapport à la rigidité oppressante des bureaux alignés des pools, des enquêtes ont montré qu'il est en même temps une source de stress liée à l'impossibilité d'avoir une zone de retrait. Il est ressenti par les employés comme une négation du territoire personnel, dans la mesure où il soumet les individus à une visibilité, une transparence et une banalisation de l'espace. Ces attitudes ont été prises en compte dans l'aménagement de nouveaux espaces de bureau basé sur la valeur du lieu personnel, abrité du regard d'autrui et créateur d'une certaine intimité.

\section{Le bureau semi-cloisonné}

Il s'agit d'un type d'aménagement basé sur le principe de cloisons mobiles qui délimitent un espace de travail sur trois côtés et qui laissent ouvert le quatrième.

L'objectif est de créer des espaces de bureaux correspondant à des groupes de travail dont les membres sont en communication directe les uns avec les autres.

Le bureau semi-cloisonné est une solution intermédiaire entre le bureau paysager et le bureau fermé. Ce bureau, appelé bureau alvéolaire ou cellulaire, peut accueillir plusieurs personnes (de 6 à 8 ou une seule). Ce type de configuration a été conçu pour permettre d'installer les employés dans un lieu ouvert tout en leur assurant un sentiment de protection et d'intimité. Son principe de base repose sur la disposition d'un type de mobilier, appelé panel system, lequel a été conçu par l'Américain Robert Probst pour la firme Herman Miller et repris en Allemagne par Modulants Elemente Program. Ici, il ne s'agit plus, comme dans le bureau paysager, de mettre tout l'environnement à contribution, mais de proposer un système d'architecture intérieure modulable par la combinaison de panneaux mobiles qui servent de supports pour le plan de travail et qui offrent en même temps un espace personnel pour le rangement. Ce type d'aménagement se présente comme un ensemble de cellules organisées en zones de travail.

La conception part de l'individu et non plus de l'espace; on joue sur le mobilier et la flexibilité pour organiser le travail. Il s'agit là d'une solution qui cherche à conjuguer l'aménagement et la communication ainsi que la concentration.

Les résultats qui se dégagent de ces différentes conceptions sont que les modèles d'aménagement fonctionnent comme des répartiteurs des activités dans le cadre du fonctionnement organisationnel; l'organisation de l'espace peut représenter un facilitateur par rapport à la circulation de l'information, mais elle n'assure pas la communication de manière automatique.

49 L'impact psychosocial de ces aménagements sur la communication montre que les individus communiquent ou non entre eux en fonction des contraintes inhérentes au travail, dont l'espace est une des expressions.

Mais parmi ces contraintes, celles liée à la structure hiérarchique sont plus déterminantes que celles liées à la structure spatiale. Donc, si l'on travaille dans une organisation qui a une structure très rigide et un aménagement très ouvert, les comportements des individus auront tendance à être plus déterminés par la structure hiérarchique ou sociale que par l'aménagement. 


\section{Modèle d'organisation du travail, nouvelles technologies et conception de l'espace}

51 La question de la conception des environnements de travail se pose depuis quelque temps en des termes nouveaux liés aux caractéristiques des nouveaux outils, c'est-à-dire des nouvelles technologies. Donc, il faut aborder le problème de l'aménagement des espaces de travail en les reliant aux nouvelles technologies et à la conception même de l'organisation et des activités.

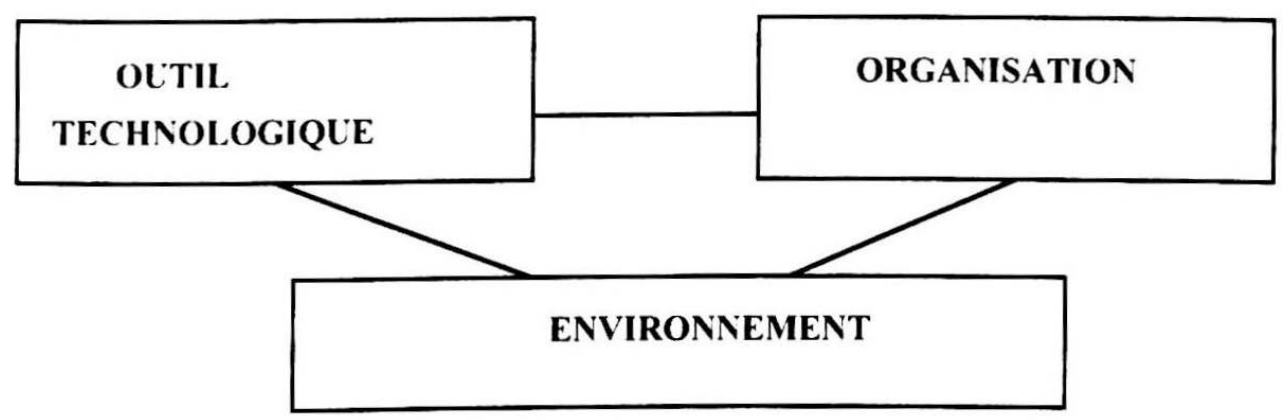

52 Depuis le début de l'ère industrielle, les modèles d'organisation n'ont jamais cessé d'évoluer vers des niveaux de complexité de plus en plus élevés et ceci notamment en raison d'un changement radical dans la manière dont circule l'information, à la fois à l'intérieur de l'entreprise, et entre l'entreprise et l'environnement.

Longtemps, l'organisation a été un univers stable. Les modèles d'informations fonctionnaient sur des processus séquentiels marqués par une logique de circulation de l'information basée sur la structure pyramidale, la centralisation des décisions et le cloisonnement des services.

Aujourd'hui, le développement technologique a entraîné des systèmes de production programmés pour permettre aux machines de traiter elles-mêmes l'information liée à certains types d'opérations.

L'informatisation des bureaux, avec l'ordinateur, fournit un potentiel en termes de source d'informations disponibles du traitement de l'information.

Les nouvelles technologies ont profondément modifié la façon de travailler et ont amené de nouvelles conceptions de l'aménagement de l'espace. Ainsi avec la bureautique, c'està-dire l'automatisation des tâches de bureau au moyen de l'informatique, on assiste à une nouvelle organisation non seulement du poste de travail, mais également de l'entreprise. Si le modèle informatique peut être considéré comme un modèle dominant aujourd'hui, son implantation dans le bureau comporte des étapes et des variantes, parmi lesquelles on peut retenir les suivantes:

La première est l'espace de bureau informatisé, qui se définit comme un système et un lieu de traitement des tâches.

58 La deuxième est l'espace de bureau automatisé, qui suppose plutôt une intégration des équipements informatiques (traitement de texte et base de données) dans une organisation informatique plus large.

59 La troisième est le bureau télématisé, lequel constitue un système où les différents postes de travail peuvent être reliés entre eux ou aux terminaux d'autres organisations. Les 
tâches sont également automatisées et il est possible de communiquer des informations à des bureaux qui possèdent eux aussi ce système. Dans ce contexte, le poste de travail traditionnel cède la place à un poste logiciel qui permet un échange d'informations entre plusieurs maillons d'un système de travail à travers un réseau informatisé.

L'informatisation des activités de bureau représente une nouvelle forme d'organisation du travail qui a entraîné une diminution du nombre d'échelons hiérarchiques, une autonomie croissante de l'employé et l'introduction extensive de techniciens et de spécialistes sur le lieu de travail. Autrement dit, les nouvelles technologies ont redéfini et redistribué la classification traditionnelle des emplois et des compétences.

Concrètement, le dispositif organisationnel se traduit par une réduction du nombre des échelons hiérarchiques et donc par un aplatissement de la pyramide sociale. On assiste ainsi, dans certains cas, et ceci de manière paradoxale, à un écart grandissant entre la « haute direction » qui prend les décisions et qui est de plus en plus enfermée dans une sphère et dans une logique de traitement des données, mais sans lien direct avec le terrain, et de l'autre, les catégories, y compris celles des cadres, qui sont des techniciens et qui, souvent, ont un haut niveau de compétence, mais auxquels on demande de devenir des animateurs de leur service, élément pour lequel ils n'ont souvent aucune formation.

La pyramide n'est donc plus seulement basée sur le seul principe hiérarchique, mais sur le rapport entre compétence et type d'apport à l'efficience organisationnelle. Il en résulte un aplatissement des niveaux hiérarchiques en trois grandes catégories : gestionnaire, innovateur, opérateur.

63 Autrement dit, dans les entreprises à haute valeur ajoutée, apparaît une dissociation entre modèle d'organisation du travail et modèle hiérarchique.

Ces changements se répercutent également sur l'espace et peuvent se traduire par une tendance à la réduction de l'espace alloué à chacun et par une augmentation des surfaces globales.

Les formes d'organisation se sont concrétisées par un remodelage de la physionomie du poste de travail et de son ergonomie : l'espace de travail, comme on l'a souligné, devient un poste logiciel intégré dans un réseau de communication; on n'a plus le même rôle de support.

66 Avec le développement de l'informatique, les contraintes matérielles liées au travail se répercutent de manière différente sur l'espace :

67 - On assiste, d'un côté, à une nouvelle configuration de l'aménagement: les activités bureautiques sont moins tributaires d'un aménagement déterminé. En d'autres termes, la question est moins de savoir dans quel type d'espace on travaille que le type de tâches qu'on accomplit.

68 - On assiste, de l'autre, à une déconnexion entre un type d'aménagement donné et la communication.

69 L'espace de travail devient un espace virtuel où l'activité dépend de moins en moins d'un lieu défini: concept d'espace portatif. On peut travailler n'importe où; loin de l'entreprise. 


\begin{tabular}{|c|c|c|l|}
\hline $\begin{array}{c}\text { Catégories } \\
\text { d'employés }\end{array}$ & $\begin{array}{c}\text { Rapport à } \\
\text { l'organisation }\end{array}$ & $\begin{array}{c}\text { Types } \\
\text { d'espaces }\end{array}$ & \\
\hline Sédentaire & Proche & & \\
\hline & $\begin{array}{c}\text { Bouge à } \\
\text { l'intérieur }\end{array}$ & Place & $\begin{array}{l}\text { Personnalisation } \\
\text { Identification }\end{array}$ \\
\hline Caboteur & Pas loin & & \\
\hline & Pas longtemps & Territoire & \\
\hline Nomade & Loin & & \\
\hline & Longtemps & Mobilité & \\
\hline
\end{tabular}

Cela entraîne de nouvelles catégories spatiales d'employés

70 En fait, on constate que l'informatique a donné lieu à de nouvelles représentations de l'espace liées à l'évolution du travail. L'aménagement de l'espace est ici le reflet d'une nouvelle représentation du travail.

71 Avec l'informatisation, l'aménagement des espaces n'est plus directement lié à la productivité ; dans un certain nombre d'entreprises, sa fonction est de servir de support à la culture de l'organisation, c'est-à-dire faciliter l'adhésion aux valeurs de l'entreprise.

72 Au début de l'informatisation, les entreprises n'ont pas innové au niveau de l'espace, mais ont imposé les changements sur les aménagements existants avec un effet de décalage entre le confort de l'espace et l'efficacité de la technologie, car les deux n'étaient pas conçus ensemble.

73 Ainsi, nombre d'entreprises ont introduit les innovations technologiques dans les aménagements existants ; cela a eu pour conséquence de créer un hiatus entre l'efficacité fournie par la technologie et le confort, car les deux n'avaient pas été conçus pour aller ensemble. Zuboff (1988) a distingué, à propos de l'introduction de l'informatique, deux processus de travail qui pouvaient être engendrés par elle : le premier est un processus qui automatise le travail (automate) en installant une technologie donnée sans remettre en cause l'organisation du travail; selon elle, il s'agit là d'une occasion manquée car l'introduction d'une nouvelle technologie est une occasion pour changer les processus; le processus automate correspond alors à la tendance à garder les mêmes aménagements, quand on introduit une nouvelle technologie. Ainsi, on installe de nouveaux ordinateurs dans une pièce, mais on ne modifie ni l'éclairage, ni la disposition des équipements. Le second est un processus qui innove (informate), c'est-à-dire dans lequel l'informatisation fournit l'occasion à la technologie de concevoir des processus organisationnels plus efficaces. Ce processus consiste à profiter de l'introduction des nouvelles technologies pour repenser les aménagements ainsi que la communication.

L'informatisation du travail a entraîné de nouveaux concepts d'aménagement.

La recherche Orbit 2 (Office Research Building and Information Technology) s'est ainsi proposée de définir les termes de l'évolution des bureaux. Cette recherche a mesuré l'impact des nouvelles technologies de l'information sur le monde du bureau. Deux idées sous-tendent cette méthode : la première concerne la typologie organisationnelle basée sur la nature du travail et la nature des changements rapides. La seconde idée est la notion de design strategy qui désigne l'ensemble de ressources physiques, assemblées pour résoudre des questions particulières d'organisation et des problèmes technologiques. 
76 Dans cette perspective, le combi-office peut illustrer le système d'aménagement conçu en fonction des nouvelles technologies; il est la combinaison de deux types d'espaces, un espace semi-cloisonné relativement petit où chacun travaille sur son ordinateur et un espace d'échanges qui est un espace ouvert plus vaste, à la disposition de chaque groupe pour les réunions et les échanges ; ce type d'aménagement cherche à satisfaire à deux exigences : la communication et la concentration nécessaire au travail sur ordinateur. Le combi-office est ainsi un concept d'aménagement qui montre un type de réponse apporté, relativement à l'espace, à une organisation informatique du travail qui suppose une flexibilité plus grande des tâches et un développement des communications.

De ce point de vue, le modèle informatique apparaît comme un système favorisant la flexibilité par :

- une dématérialisation des activités avec une circulation et une maîtrise des informations ; des échanges et la création de réseaux ;

79 - une activité qui n'est plus dépendante d'un lieu, mais devient un poste logiciel dans un réseau de communication ;

80 - une activité qui dépend de l'accès à l'information et du traitement qui en est fait, en temps réel, d'où des activités discontinues dans des lieux différents: accessibilité, création, communication.

81 Autrement dit, ce qui devient important, c'est le traitement de l'information et l'instantanéité des données recueillies. On passe d'un concept spatial à un concept temporel dans lequel le lointain devient proche; ce qui entraîne un aplatissement de la distance et de l'espace.

82 L'évolution de l'organisation et des activités, liée aux outils informatiques, fait apparaître deux critères nouveaux qui déterminent le travail : le niveau d'interaction et le niveau d'autonomie.

83 En fonction de ces deux critères, on peut identifier des types d'organisation où le rapport autonomie-interaction est faible et d'autres où il est élevé. II apparaît que c'est dans les espaces où ce rapport est le plus élevé que la conception des nouveaux espaces semble le plus se développer et est la plus adaptée.

84 Dans cette perspective, la relation entre nouveaux outils informatiques et nouvelle conception des aménagements apparaît dans des secteurs d'activités où le rapport interaction-autonomie est le plus élevé au niveau des activités. Le schéma 1 suivant présente une répartition des types d'entreprises ou de secteurs d'activités selon ces critères. 


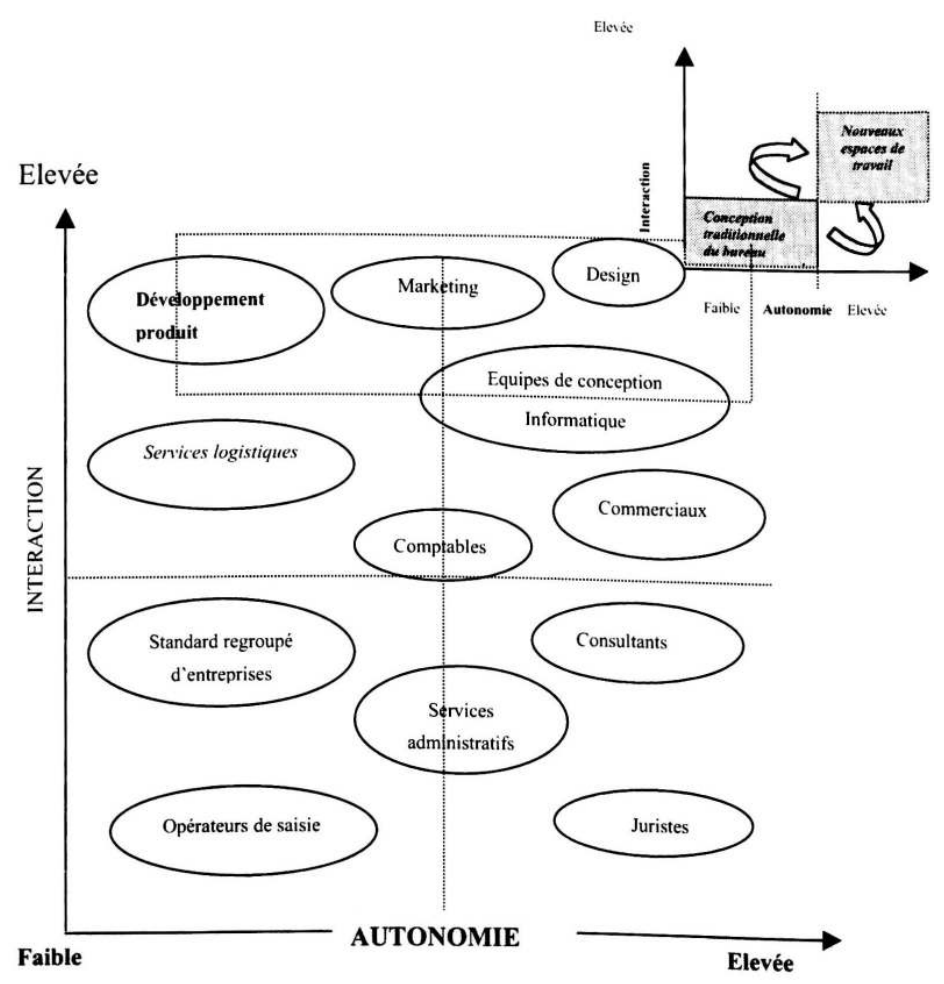

Schèma 1

Source: Steelcase Strafor

\section{Application : le working lunch}

Pour illustrer la problématique des recherches et des projets d'aménagement liés à l'évolution technologique, nous présenterons une démarche appelée « Working Lunch ».

\section{Présentation}

Deux entreprises, Steelcase Europe et Alcatel, se sont associées et ont travaillé pendant un an avec le cabinet de design prospectif américain IDEO.

Leur projet, appelé working lunch, a consisté à rechercher le type d'environnement le plus adéquat par rapport aux nouveaux outils technologiques; c'est l'idée de restaurant qui s'est imposée dans leur démarche comme la plus pertinente par rapport aux nouvelles activités et comme nouveau lieu de travail. Pourquoi le restaurant? Parce que les activités de bureau qui vont se développer sont celles qui, à leurs yeux, correspondent le plus au comportement adopté dans un restaurant.

Autrement dit, la nouvelle conception de l'espace de travail repose sur le principe suivant : « On ira au travail comme on irait au restaurant ».

Le concept du restaurant apparaît comme le plus approprié parce qu'il offre une dynamique du setting basée sur deux éléments fondamentaux : le menu et le service. 

rojet intègre donc dans le cadre professionnel des éléments issus du restaurant en proposant à la fois le mobilier et les outils, l'environnement et les services qui s'adaptent le mieux aux nouveaux modes de travail.

91 Dans le restaurant, six opportunités d'aménagement par rapport au bureau ont ainsi été identifiées : buffet, bar, salon, menu, table, cabine téléphonique, (cf. schéma 2).

buffet
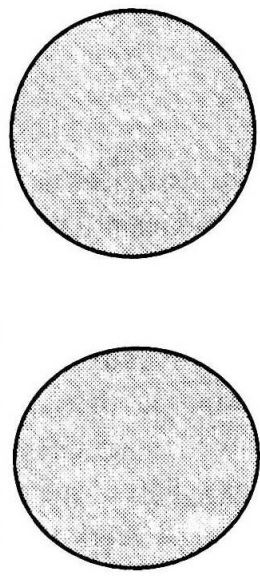

today specials har
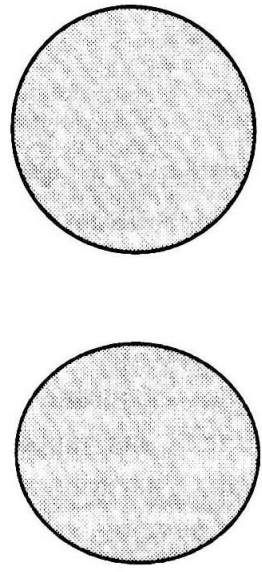

table dining salon
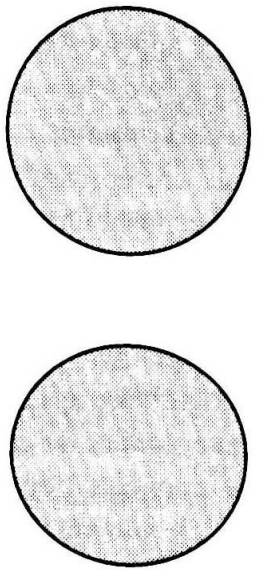

phone box

\section{6 design opportunities}

Schéma 2

Source: Steelcase Strafor

92 Ces six espaces, qui sont le support du working lunch, sont distribués selon deux axes : un axe espace privé/espace public et un axe travail personnel/travail partagé. La combinaison de ces deux axes avec les six types d'espace constitue une nouvelle vision de l'environnement de bureau (cf. schéma 3). 


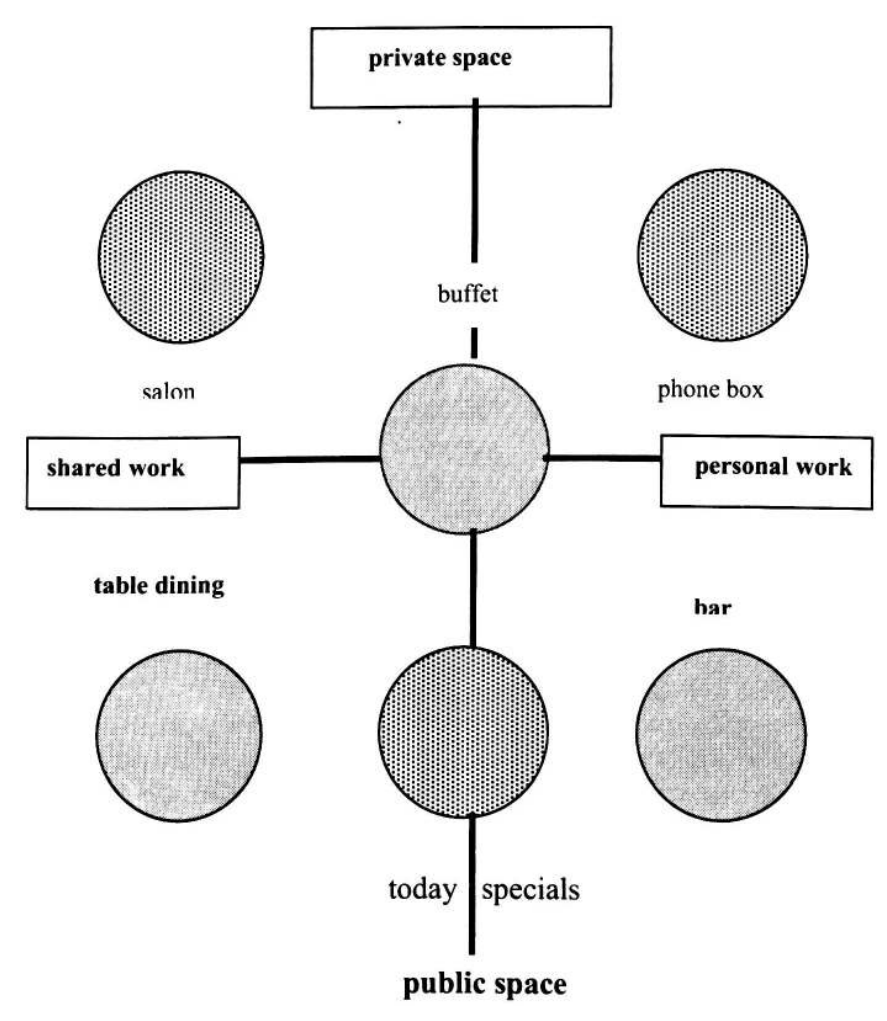

Schéma 3

Source : Steelcase Strafor

\section{Fonctionnement}

$93 \mathrm{Si}$ l'on considère le fonctionnement et l'activité des individus dans ce nouvel environnement, on observe qu'à ce type d'environnement correspondent de nouveaux types d'outils essentiellement informatiques, mais dont la caractéristique commune est qu'ils sont tous portables, c'est-à-dire qu'ils ne nécessitent plus aucune infrastructure câblée.

Chaque employé possède ainsi trois types d'outils :

- Un C zam (sésame), c'est-à-dire un badge qui a une forte caractéristique personnelle et qui lui indique qui est dans l'entreprise, où et ce qu'il y fait.

- Un magie pen pour écrire sur toutes les surfaces.

- Un écran interactif sans fil qui remplace l'ordinateur actuel.

Concrètement, le processus du travail se déroule de la manière suivante :

À son arrivée dans l'entreprise, un panneau interactif, le menu, présente des informations internes ou externes à l'employé.

Celui-ci se rend ensuite au buffet où il prend un plateau sur lequel il pose tous les outils dont il aura besoin dans la journée : écran interactif.

Il peut ensuite se diriger vers le bar, lieu de convivialité temporaire, équipé de copieurs, scanners, fax ; c'est l'endroit idéal pour des tâches de courte durée. 
Pour des tâches de longue durée, c'est l'espace table, conçu comme un box de restaurant, qui permet d'accueillir quatre à six personnes pour travailler à une activité de groupe ou à un projet.

Le salon est un lieu pour des présentations plus formelles ; il est aménagé sous forme d'un module équipé d'écrans et où chaque élément peut s'organiser et se ranger selon le bon vouloir des usagers. Cet endroit est équipé de chaises avec écrans.

4 Enfin, pour s'isoler, des phone boxsont installés, sur le modèle des cabines téléphoniques; ils résolvent le problème des conversations privées par téléphone. Le tabouret peut être utilisé comme siège ou plan de travail dans la cabine même.

5 Tout l'équipement et le mobilier (schéma 4) ont été conçus pour être suffisamment flexibles et adaptables, et ceci en vue de répondre à une double exigence : d'une part, au besoin de privatisation, et de l'autre, à celui de coopération et de travail en équipes.

6 L'aménagement intérieur repose ainsi sur une logique de composants interdépendants délimités par une structure métallique qui peut être fermée ou ouverte, suivant les besoins d'une activité. Ces modules sont adaptables à volonté en fonction des activités de chaque entreprise car ils sont parfaitement indépendants de la structure du bâtiment.

7 Les données fournies par le projet Working Lunch mettent en évidence plusieurs caractéristiques et enjeux de l'évolution dans la conception des aménagements. Elles indiquent en quels termes nouveaux se poseront les relations entre espace et communication au niveau du travail.

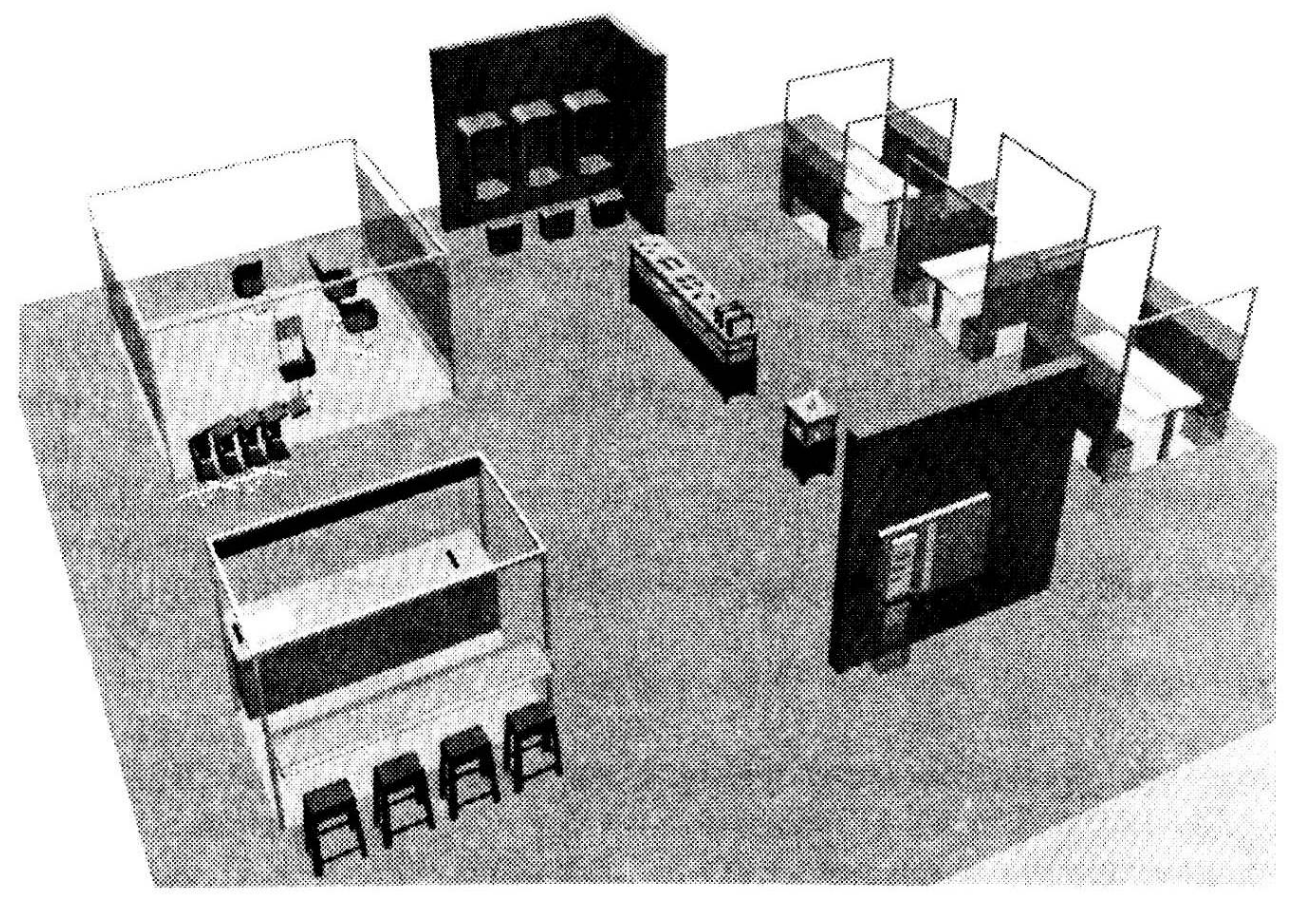

Schéma 4

Source : Steelcase Strafor 


\section{Conclusion}

108

Les environnements de travail, les nouveaux outils technologiques et l'organisation constituent un système d'interdépendance; on ne peut pas les aborder et les traiter séparément. Il faut en effet toujours considérer la dynamique existant entre eux et notamment les relations entre modification de l'espace, nouveau projet d'aménagement et fonctionnement organisationnel.

De manière plus précise, en ce qui concerne les démarches et conceptions d'aménagement, c'est la manière d'y arriver qui est importante ; la valeur d'une solution est dans le chemin qu'on prend pour y parvenir. Il importe donc que les lieux de travail soient l'objet d'une évaluation qualitative afin de mieux comprendre les relations entre le comportement et la psychologie humaine et de déterminer les effets créés par le contexte environnemental au sein duquel on travaille.

Dans cette perspective, les nouveaux environnements de travail posent la question de savoir comment on veut faire travailler les individus dans la mesure où l'on introduit de nouvelles modalités d'intégration et de socialisation professionnelle et où la valeur de l'espace comme territoire personnel et de groupe est redéfinie.

La conception proposée par Working Lunch en est une démonstration; elle ne repose plus sur une vision essentiellement "ergonomique » du travail, basée sur une adéquation optimale de l'outil et de l'opérateur, mais sur une compréhension globale de nouvelles données qui déterminent l'activité, à savoir notamment les outils technologiques.

\section{BIBLIOGRAPHIE}

BARKER, R.G., Ecological Psychology: Concepts and Methods for Studying the Environment of Human Behavior. Standford (Cal.): Stanford University Press, 1968.

CALHOUN, J.-B. Population density and Social Pathology. Scientific American, 1962. $\mathrm{n}^{\circ} 206$, p. 139-146.

DAVIS, T.M.R., The Influence of the Physical Environment in Offices. Academy of Management Review. 1984. t. 9, nº 2, p. 271-283. EVETTE, T.. LAUTIER, F. (dir.), De l'atelier au territoire : le travail en quête d'espaces. Paris : L'Harmattan., 1994.

FISCHER, G.N., Le bureau, espace de la vie quotidienne. In L'empire du bureau. Paris : BergerLevrault, 1984.

FISCHER, G.N., Psychologie des espaces de travail. Paris : Armand Colin. 1989.

FISCHER, G.N., Psychologie des Arbeitsraumes. Frankfurt/Main : Campus Verlag/Paris : Ed. de la Maison des Sciences de l'Homme, 1990. FISCHER, G.N., Les concepts fondamentaux de la psychologie sociale. Paris : Dunod/Montréal, Les Presses de l'Université de Montréal, 1996. 2è éd.

FISCHER, G.N., Individuals and Environment. À Psychosocial Approach to Workspace. Berlin : Walterde Gruyter, 1997. 
FISCHER, G.N., Psychologie de l'environnement social. Paris : Dunod/Bo-Pré : Québec, 1997, 2è éd.

FISCHER. G.N.. Travail et vie sociale. In La Psychologie Sociale. Paris : Le Seuil. 1997. p. 331-370.

FISCHER, G.N., L'évaluation des environnements de travail : approche théorique et méthodes. Psychologie Française. 1997, t. 42, n² 2, p. 139-147. FISCHER. G.N. \& VISCHER, J.-C, L 'évaluation des environnements de travail. La méthode diagnostique. Montréal : Les Presses de l'Université de Montréal/Bruxelles, De Boeck Université, 1998.

FISCHER, G.N. \& TARQUINIO, C, Schéma de soi et perception des environnements de travail. Psychologie du Travail et des Organisations. 2000, t. 6, n 1-2, p. 23-43.

GOODRICH, R., Seven Office Evaluations. Environment and Behavior. 1982, t. 14, n 3, p. 353-378.

HALL, E.T., Le langage silencieux. Paris: Marne. 1973. HEDGE, A., Design Innovations in Office Environments. In PREISER, W., VISCHER, J., WHITE, E. (Eds.). Design Intervention : Toward a More Humane Architecture. New York: Van Nostrand Reinhold, 1982, p. 301. LAUTIER, F., Ergotopiques. Toulouse: Octares Editions, 1999.

OSMOND, H., The Relationship between Architect and Psychiatrist. In GOSHEN, C. Psychiatric Architectur. Washington D.C.: American Psychiatric Association, 1959.

SUNDSTROM, E. \& SUNDSTROM, M.G., Work Places: The Psychology of the Physical Environment in Offices and Factories. New York: Cambridge University Press, 1986.

VISCHER, J.-C, Workspace Strategies: Environment As a Tool for Work. New York: Chapman and Hall, 1996.

ZUBOFF, S.. In the Age of the Smart Machine : The Future of Work and Power. New York : Basic Books, 1988.

\section{RÉSUMÉS}

Le texte présente une approche psychosociale des espaces de travail. il est plus particulièrement centré sur les impacts des aménagements et considère les interactions existant entre l'organisation des environnements et la satisfaction ainsi que l'efficacité au travail. Après une présentation des principaux modèles d'aménagement et d'organisation du travail liés en particulier aux nouvelles technologies, nous montrerons les formes d'interactions existant entre outils technologiques, organisation et environnement. Pour illustrer ces différentes dimensions, nous présenterons une démarche d'application.

This paper presents the psychosocial approach of workspaces; it is organized around the major concepts of environmental psychology and the models of organizations and environmental design. It analyzes the interactions and their impacts on the productivity and the psychological comfort. An application offers tools that can contribute to creating sucessful working conditions by new technological workspaces.

\section{INDEX}

Mots-clés : territoires, impact psychosocial, aménagement, nouvelles technologies, psychologie de l'environnement 


\section{AUTEURS}

\section{GUSTAVE-NICOLAS FISCHER}

Gustave-Nicolas Fischer est professeur de psychologie sociale ; il est directeur du laboratoire de psychologie à l'université de Metz et professeur invité dans plusieurs universités étrangères (Montréal, Lisbonne, Lausanne). Après avoir été durant plusieurs années psychologue du travail et consultant dans les entreprises, il a développé ses recherches sur la psychologie des environnements de travail en étudiant l'impact psychosocial des aménagements sur les employés. Il a élaboré, en collaboration avec une chercheuse du M.I.T., une méthodologie d'évaluation des environnements de travail.

\section{CHANTAL FOUSSE}

Chantal Fousse est assistante de recherche au laboratoire de psychologie de l'université de Metz. Après avoir travaillé de nombreuses années dans le secteur privé, elle collabore aux recherches en psychologie de l'environnement au laboratoire de psychologie. 